\title{
Are EDS Specifications Still Relevant
}

\section{Keith Thompson ${ }^{1}$ \\ 1. Thermo Fisher Scientific, Madison, WI USA}

The spectral performance of an EDS detector is almost universally described by measuring the Mn kalpha line at an x-ray collection rate of a few thousand counts per second. In many cases, this is the only metric cited in describing detector performance. The rationale is mainly historic and based on the ready availability of $\mathrm{Fe}^{55}$ as an inexpensive and relatively weak (thereby safe) source of radiation emitted at the Mn-k line. This specification presented a convenient and pragmatic metric for LN-cooled Si(Li)based EDS detectors whose Be window occluded most of the low energy portion of the spectrum. In an era of modern, SDD-based EDS detectors with high count rates and light element windows, the use of this simplified specification warrants examination.

Table 1 quantifies the impact of monitoring the low energy section of the spectrum relative to measuring only the Mn k-line. The average energy resolution for several x-ray energy lines is tabulated for two unique EDS detector designs. 200 EDS detectors were tested in this study. The $\mathrm{x}$-ray collection rate was 5,000 counts per second. Both $30 \mathrm{~mm}^{2}$ and $60 \mathrm{~mm}^{2}$ active area detectors were examined. The 1 sigma variation within each group was roughly $1.3 \mathrm{eV}$. Design Group-1 was optimized for low energy performance. Design Group-2 was not optimized for low energy performance. At the Mn k-line, both the $30 \mathrm{~mm}^{2}$ and $60 \mathrm{~mm}^{2}$ active area detector groups report energy resolution values within $\sim 2-3 \mathrm{eV}$ of each other. While these groups are statistically different at a $95 \%$ confidence interval, the difference in spectral quality is not discernible when the spectra are overlaid. The light element results $(<1000 \mathrm{eV})$ provide a very different result. The difference in energy resolution between the two design groups is roughly $10 \mathrm{eV}$; a very substantial improvement in performance relative to the already low $(\sim 60 \mathrm{eV})$ energy resolution in this part of the spectrum. This performance improvement is seen for both the 30 $\mathrm{mm}^{2}$ and the $60 \mathrm{~mm}^{2}$ active area detectors. While both detector groups qualify for a sub-130 eV energy resolution rating under the traditional Mn-k specification, the actual difference in performance between the two design groups is substantial.

This difference in performance can be visualized by overlaying the spectrum. Figure 1 shows the spectra acquired from a group-2 EDS detector at count rates of 10,000 input (9,500 output) counts per second and at 1,000,000 input (300,000 output) counts per second. Visual inspection demonstrates that the spectrum at Mn k-alpha is only moderately degraded at the substantially higher count rate. Inspection of the low energy spectrum (magnified for clarity) realizes a dramatic change in spectral quality. At only 10,000 counts per second, the Mn-L and O-k lines are clearly distinguishable. At 1,000,000 counts per second these lines overlap to form a single, broad peak.

The benefit to the low energy part of the spectrum with increasing count rate for an optimized detector (Group-1) is demonstrated in Figs. 2 and 3. Figure 2 displays energy spectra with B, C and $\mathrm{O}$ at both 10,000 and 100,000 counts per second. The spectral quality of the B and C peaks are only slightly degraded between the two count rates, and the peaks are still well separated. Figure 3 displays the energy spectrum of an Alumina Silicate sample, containing Si Al and O. Once again, there is no discernible degradation in spectral quality between 10,000 and 300,000 counts per second. There is 
some degradation in the spectrum at 1,000,000 counts per second. However, the peaks are still cleanly resolved and separable.

The universally accepted specification of Mn k-alpha measured at only a few thousand counts per second fails to properly describe the performance of a modern EDS detector. Because of its broad acceptance, this metric grossly misrepresents the overall EDS performance. A properly optimized EDS detector should perform consistently well throughout the entire energy spectrum and at both low and high $(>100,000)$ x-ray collection rates. A modern EDS detector specification should reflect this requirement by additionally specifying performance at a light element $x$-ray line (e.g. F) and at a high collection and conversion rate (e.g. $>100,000$ counts per second at $<50 \%$ dead time).

Table 1.

Energy resolution $(\mathrm{eV})$ measured at several X-ray lines for 2 distinct EDS detector designs.

\begin{tabular}{|c|c|c|c|c|}
\hline EDS deisgn group & Mn k & Si k & F & CK \\
\hline \multicolumn{5}{|c|}{} \\
\hline $30 \mathrm{~mm}$ 2 - group 1 & 126.5 & 77.6 & 60.4 & 51.8 \\
\hline $30 \mathrm{~mm}$ 2 - group 2 & 129.6 & 84.7 & 70.5 & 62.4 \\
\hline \multicolumn{5}{|c|}{} \\
\hline $60 \mathrm{~mm}$ - group 1 & 125.8 & 76.8 & 57.6 & 48.9 \\
\hline $60 \mathrm{~mm}$ 2 - group 2 & 127.4 & 81.9 & 67.3 & 59.1 \\
\hline \hline
\end{tabular}

Figure 1 (right). Mn spectrum from a design group 2 EDS detector at 10,000 and 1,000,000 input counts per second.

Figure 2 (bottom left). B C O spectrum from a design group-1 detector at 10,000 and 100,000 input counts per second (DT <30\%)

Figure 3 (bottom right). Si Al O spectrum from a design group-1 detector at 10,000,300,000, and $1,000,000$ input counts per second (DT $<50 \%$ )
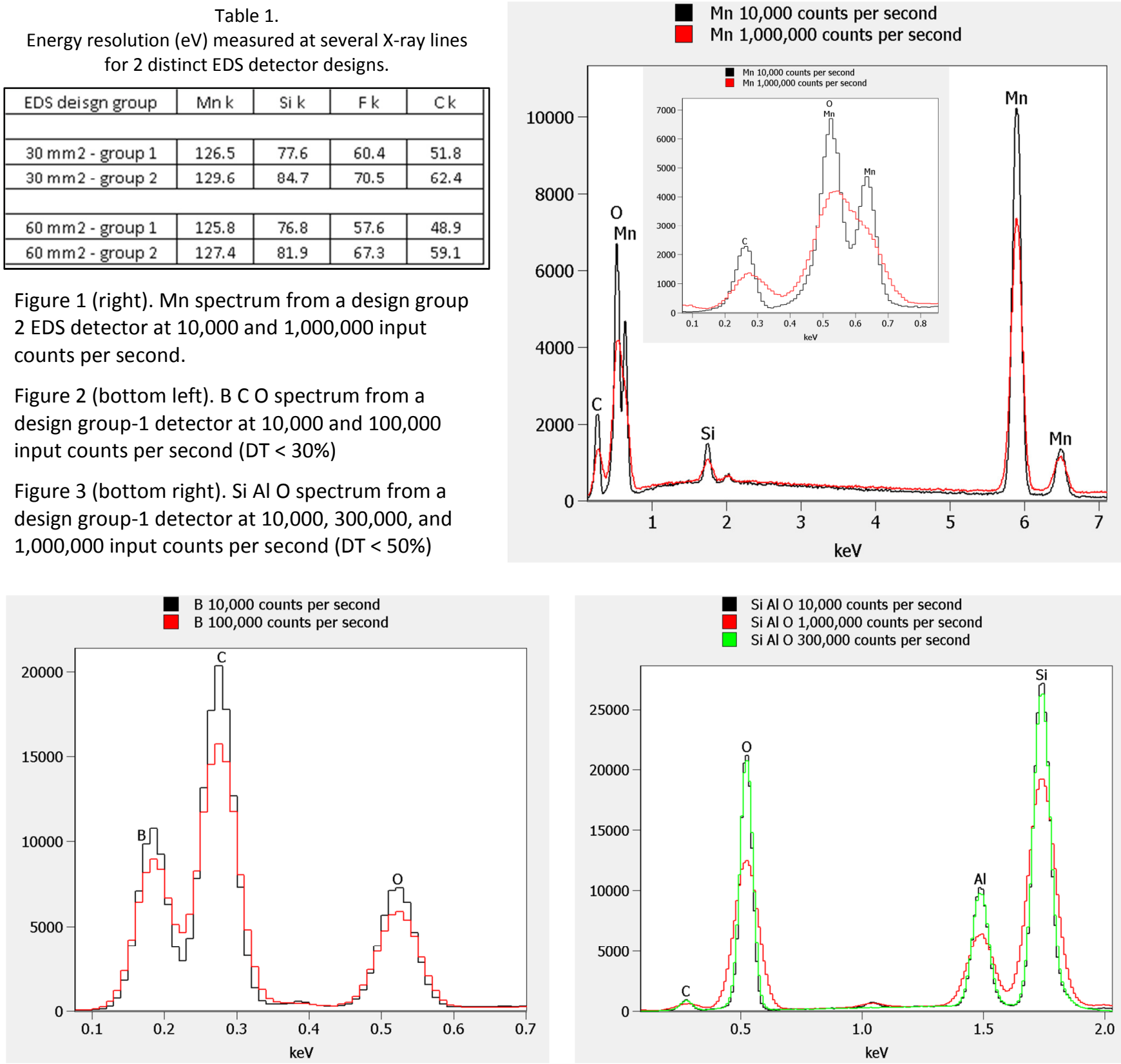

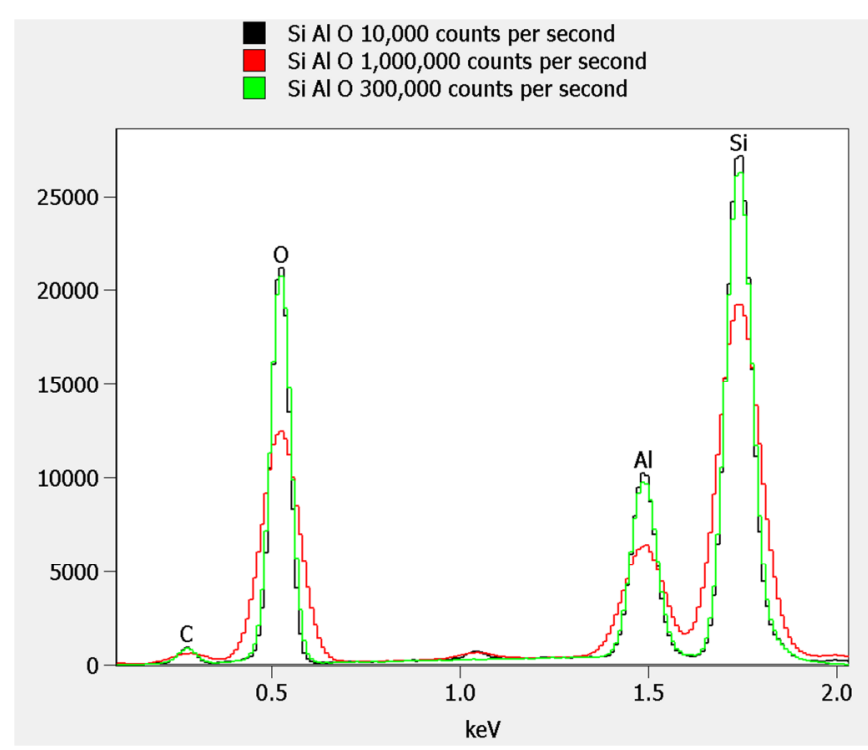

